\title{
Essai de valorisation agricole de déchets industriels contenant de l'azote nitreux et nitrique
}

\author{
B Lagacherie *, Y Couton, JC Germon \\ INRA, laboratoire de microbiologie des sols, 17 rue Sully BV 1540, 21034 Dijon cedex
}

(Reçu le 20 septembre 1992; accepté le 4 décembre 1992)

\begin{abstract}
Résumé - Deux déchets industriels contenant 10-15\% d'azote, moitié sous forme nitreuse et moitié sous forme nitrique, ont servi de source d'azote à des cultures de blé et de maïs. En incubation de laboratoire, la conversion des nitrites en nitrates varie de 22 à $52{\mathrm{mg} . \mathrm{kg}^{-1} \text {.semaine }}^{-1}$ d'azote en sol neutre pour des températures de 10,15 et $20{ }^{\circ} \mathrm{C}$; en sol acide au moins un tiers de l'azote nitreux est perdu par dénitrification chimique et le fonctionnement d'une partie de la microflore semble perturbé : minéralisation accrue du carbone et production d'ammonium. Au champ la vitesse de conversion est conforme aux résultats de laboratoire lorsque le déchet est apporté en solution mais diminue de moitié avec les produits solides. La persistance du nitrite dans le sol est fonction de la dose apportée. Un apport sur blé à la montaison pénalise légèrement le rendement en grain $(-5 \%)$, les nitrites ralentissant l'assimilation azotée des plantes. Sur maïs au semis la germination est fortement perturbée par les nitrites, entraînant des diminutions de rendement en grain pouvant atteindre $30 \%$ selon la dose d'apport. Lorsque la température du sol atteint $10^{\circ} \mathrm{C}$ un apport de $50 \mathrm{~kg} \cdot \mathrm{ha}^{-1}$ d'azote nitreux sur culture implantée (blé) permettrait la valorisation de ce type de déchets par la voie agricole.
\end{abstract}

\section{déchet / nitrite / microflore / azote / sol}

Summary - Trial on agricultural use of industrial waste containing nitrous and nitric nitrogen. Two batches of industrial waste containing 10-15\% oxidized inorganic $N$, half of which was in nitrite form, were used as $N$ source for wheat and maize crops. In laboratory incubation the conversion of nitrite to nitrate varied from $22-52 \mathrm{mg} \mathrm{N} . \mathrm{kg}$ soir ${ }^{1}$ $w k^{-1}$ at temperatures of 10,15 and $20^{\circ} \mathrm{C}$ in soil with a neutral $\mathrm{pH}$. In acid soil at least one-third of the nitrite $\mathrm{N}$ was lost by chemical denitrification and the functioning of part of the microflora appeared to be perturbed: there was an increased mineralization of $C$ and production of ammonia. In the field, the rate of conversion corresponded with laboratory results when the waste was applied as a solution, but halved with solid products. Persistance of nitrite in the soil was a function of the dose added. Application to wheat at the beginning of stem elongation slightly reduced grain yield $(-5 \%)$, with nitrites slowing down $N$ assimilation of the plants. Applied to maize at sowing, germination was strongly affected by nitrites, resulting in a diminution of grain yield which reached $30 \%$ depending on the application dose. When soil temperature had attained $10^{\circ} \mathrm{C}$, an application of $50 \mathrm{~kg} \cdot \mathrm{ha}^{-1}$ of oxidized $\mathrm{N}$ to the growing crop (wheat) allowed the 2 batches of waste to be used in agriculture.

waste / nitrite / microflora / nitrogen / soil

\section{INTRODUCTION}

Le sol est parfois utilisé pour épurer des déchets industriels (Germon, 1985; Bourquin, 1990). Ce sont souvent des solutionssuspensions aqueuses; certains des composants (azote, eau) participent à la formation de la biomasse végétale, conférant ainsi au déchet une valeur agronomique. Pour certains déchets l'élimination est plus délicate : ainsi les mélanges de nitrates et de nitrites de potassium et de sodium, utilisés dans l'industrie comme agents caloporteurs ou sels de bains de trempe (métallurgie de surface des aciers spéciaux), et dont la consommation française est de l'ordre de 5 à 10000 T.an $^{-1}{ }^{\star \star}$. Actuellement ces pro-

\footnotetext{
* Correspondance et tirés à part

** Source Anred, 2 square Lafayette, 49000 Angers
} 
duits sont concentrés dans des décharges contrôlées où ils représentent un risque pour l'environnement.

Si la présence d'azote nitrique se traduit par une valeur fertilisante azotée initiale, la présence d'azote nitreux, en quantité équivalente, constitue un réel problème. L'utilisation d'azote nitreux comme fertilisant a été envisagée et expérimentée dès 1905 (Lenglen, 1947) : à des doses de $50 \mathrm{~kg}$ d'azote apporté par ha l'efficacité fertilisante est équivalente à celle de la forme nitrique. Van den Hende (1950) observe des dégâts (manques à la levée, brulûres de feuillage) sur certaines espèces cultivées, pour des doses de $100-160 \mathrm{~kg}$ d'azote ha $^{-1}$ et suggère une utilisation des sels nitreux comme désherbant. Si l'ion nitreux peut être directement assimilé et utilisé par la plante (Lemaire, 1969), il se révèle souvent toxique (Paul et Polle, 1965; Oke, 1966) dès que la concentration en azote nitreux atteint $50 \mathrm{mg} \cdot \mathrm{kg}^{-1}$ de sol dans la rhizosphère (Goyal et Huffaker, 1982), cette toxicité étant renforcée à $\mathrm{pH}$ bas. II constitue également l'un des intermédiaires de la nitrification et la plante peut l'utiliser après transformation en azote nitrique. Le passage nitrite-nitrate est toutefois sous la dépendance de certains facteurs pédoclimatiques : dans un sol à $\mathrm{pH}$ bas, riche en matière organique, la dénitrification chimique peut être favorisée (Nelson et Bremner, 1969; Van Cleemput et Baert, 1984); en sol alcalin, les basses températures, une forte concentration en ammonium, freinent l'oxydation de l'ion nitreux (Chapman et Liebig, 1952; Tyler et al, 1959). Ce ralentissement est attribué à la présence d'ammoniac libre qui exerce une action inhibitrice sur Nitrobacter, en milieu acide l'acide nitreux non dissocié exercerait un effet semblable (Anthonisen et al, 1976).

Le sol pourrait jouer un rôle épurateur pour des déchets contenant de l'azote nitreux et permettre de valoriser l'azote qu'ils contiennent par une culture à condition que certains préalables soient levés : choix du sol de la parcelle d'épandage, de la culture et du stade d'apport, de la dose d'apport. Afin de répondre à d'éventuels utilisateurs de ces déchets, nous avons cherché à préciser les risques encourus et les moyens de les minimiser. Ceci nous a conduits à examiner en laboratoire la transformation de l'azote nitreux de 2 types de déchets, en prenant en compte les effets liés au $\mathrm{pH}$ du sol, à la concentration de produit apporté et à la température. Des effets plus globaux sur culture déjà implantée (blé à la montaison) ou au moment de la levée (maïs) ont ensuite été étudiés au champ afin d'étudier la faisabilité de l'épandage; ces observations au champ ont été complétées par une expérimentation en serre destinée à apprécier et à expliquer certaines perturbations observées lors de la levée, et prenant notamment en compte les modalités de l'épandage (forme solide ou après mise en solution et neutralisation).

\section{MATÉRIEL ET MÉTHODES}

\section{Caractérisation des déchets}

Les 2 déchets, obtenus par vidange des circuits calorifiques (déchet $A$ ) ou écrémage des bains de trempe (déchet B) prennent en masse lors du refroidissement et se présentent sous la forme de blocs de sels très durs. Outre l'azote, nous avons étudié un certain nombre d'éléments potentiellement dangereux : métaux lourds, cyanures et baryum, compte-tenu de leur importance pondérale dans le déchet $B$ et de leur potentielle toxicité (Maillard, 1958).

Les déchets énergiquement concassés puis broyés ont été analysés par le laboratoire d'analyse des sols de I'INRA à Arras (après attaque fluorhydrique pour les formes solides). Les cyanures totaux ont été dosés par le laboratoire d'analyse d'eau de la ville de Paris (méthode NF T 90 107). L'azote nitreux et nitrique a été dosé par notre laboratoire, sur solution obtenue par épuisement de l'échantillon à l'eau, en flux continu par adaptation de la méthode de Griess-losvay (Nicolardot, 1988).

\section{Caractéristiques des sols}

Les sols utilisés lors de cette étude ont été analysés par le laboratoire d'analyse des sols de l'INRA à Arras (terre fine de l'horizon 0-20 cm). Leurs principales caractéristiques sont indiquées dans le tableau $\mathrm{I}$.

Les sols 1 et 4 sont des sols bruns calcaires de la plaine dijonnaise; les sols 3 et 5 , issus de cette même plaine, sont des sols bruns calciques. Le sol 2 provient d'une arène granitique en bordure du Morvan.

\section{Incubations de laboratoire}

L'évolution de l'azote minéral a été suivie dans 2 sols différents (sol $1 \mathrm{pH}=7,9$ et $2 \mathrm{pH}=4,6$ du tableau I) non amendés (témoins), recevant 4 doses du déchet B (correspondant à 30, 60, 90 et $120 \mathrm{mg}$ d'azote nitreux par $\mathrm{kg}$ de sol sec appliqué sous forme de solution aqueuse) ou les sels de référence $(60 \mathrm{mg} \mathrm{N}$ $\mathrm{KNO}_{2}$, ou $\left.60 \mathrm{mg} \mathrm{N}-\left(\mathrm{NH}_{4}\right)_{2} \mathrm{SO}_{4}\right)$. L'incubation a eu lieu 
Tableau I. Caractéristiques physico-chimiques des sols ayant servi pour cette étude.

\begin{tabular}{|c|c|c|c|c|c|}
\hline & $\begin{array}{c}\text { Sol } 1 \\
\text { Incubation } \\
\text { labo }\end{array}$ & $\begin{array}{c}\text { Sol } 2 \\
\text { Incubation } \\
\text { labo }\end{array}$ & $\begin{array}{l}\text { Sol } 3 \\
\text { Blé } \\
\text { champ }\end{array}$ & $\begin{array}{l}\text { Sol } 4 \\
\text { Maïs } \\
\text { champ }\end{array}$ & $\begin{array}{l}\text { Sol } 5 \\
\text { Mais } \\
\text { serre }\end{array}$ \\
\hline \multicolumn{6}{|l|}{ Granulométrie $\left(\mathrm{g} \cdot \mathrm{kg}^{-1}\right)$} \\
\hline argiles & 343 & 191 & 267 & 402 & 334 \\
\hline limons fins & 251 & 148 & 372 & 293 & 322 \\
\hline limons grossiers & 173 & 81 & 278 & 229 & 219 \\
\hline sables fins & 179 & 126 & 54 & 50 & 101 \\
\hline sables grossiers & 54 & 454 & 29 & 26 & 24 \\
\hline C organique $\left(\mathrm{g} \cdot \mathrm{kg}^{-1}\right)$ & 15,3 & 24,5 & 12,2 & 13,3 & 12,0 \\
\hline N Kjeldahl $\left(\mathrm{g} \cdot \mathrm{kg}^{-1}\right)$ & 1,8 & 2,2 & 1,2 & 1,7 & 1,4 \\
\hline $\mathrm{C} / \mathrm{N}$ & 8,5 & 10,9 & 10,6 & 7,8 & 8,6 \\
\hline Calcaire total $\left(\mathrm{g} \cdot \mathrm{kg}^{-1}\right)$ & 17 & 0 & 0 & 6 & 2 \\
\hline CEC (méq. $100 \mathrm{~g}^{-1}$ ) & 21,5 & 11,8 & 13,6 & 21,0 & 20,0 \\
\hline $\mathrm{pH}$ eau & 7,9 & 4,6 & 6,6 & 7,2 & 7,2 \\
\hline Humidité équiv (g.100 $\left.\mathrm{g}^{-1}\right)$ & 25,3 & 21,6 & 23,6 & 25,6 & 24,6 \\
\hline
\end{tabular}

à $20^{\circ} \mathrm{C}$, l'humidité des sols étant maintenue à humidité équivalente.

L'évolution de l'azote minéral a également été suivie dans le sol 1 ayant reçu une dose du déchet $B$ apportant $60 \mathrm{mg}$ d'azote nitreux par $\mathrm{kg}$ de sol. L'incubation a été réalisée à 3 températures : 10,15 et $20^{\circ} \mathrm{C}$, l'humidité du sol étant maintenue à $80 \%$ de l'humidité équivalente.

La source d'azote nitreux utilisée dans ces incubations est le lot 1 du déchet $B$ (tableau $V$ ). Les échantillons correspondaient à $100 \mathrm{~g}$ de sol sec placés dans des fioles à plasma de $575 \mathrm{~cm}^{3}$ de volume, fermées, le dioxyde de carbone produit étant récupéré par un piège contenant $10 \mathrm{~cm}^{3}$ de $\mathrm{NaOH} \mathrm{0,2} \mathrm{N}$. Chaque semaine les pièges à $\mathrm{CO}_{2}$ étaient renouvelés et 4 échantillons par traitement sacrifiés pour déterminer l'azote minéral. Le dioxyde de carbone était dosé par colorimétrie en flux continu (Chaussod et al, 1986a). L'azote minéral, extrait par une solution normale de $\mathrm{KCl}$ (rapport poids sol/volume solution : $1 / 5$ - agitation $1 \mathrm{~h}$ à $20^{\circ} \mathrm{C}$ ) a été dosé selon la méthode déjà décrite pour les formes nitreuse et nitrique, et par colorimétrie en flux continu selon la réaction de Berthelot (Nicolardot, 1988) pour la forme ammoniacale. La production de protoxyde d'azote a été mesurée dans l'atmosphère des fioles d'incubation par analyse d'une aliquote (CPG colonne acier $1 / 82 \mathrm{~m}$, Porapak $Q$, four $40^{\circ} \mathrm{C}$, argonméthane $90-10$, détecteur à capture d'électrons $63 \mathrm{Ni}$ ).

\section{Essais de culture}

Au champ, nous avons suivi l'oxydation de l'azote nitreux dans le sol sous 2 cultures, et l'effet de l'apport des déchets (solides ou en solution neutralisée) sous plante cultivée, ces apports étant effectués sur cultures en végétation (blé) ou en post-semis (maïs). Nous avons apprécié leur efficacité fertilisante globale en comparant les quantités de grains récoltées à celles d'un témoin ayant reçu une fertilisation azotée classique (ammonitrate) complétée d'un apport de potassium.

En serre, nous avons mesuré l'incidence des formes d'apport du déchet B (solide, en solution) sur la levée et les premiers stades végétatifs du maïs.

\section{Essais au champ}

Pour chaque plante, un essai en blocs à 4 répétitions a été mis en place (sols 3 et 4 , tableau I) : les principales modalités sont résumées dans le tableau II et les traitements récapitulés dans le tableau III.

Les épandages d'engrais et de déchet solide ont été réalisés manuellement; les déchets ont d'abord été cassés à la masse puis broyés (diamètre $5 \mathrm{~mm}$ ) à l'aide d'un broyeur à marteaux (blé) ou grossièrement pour diminuer la fraction pulvérulente (maïs). Les épandages des solutions liquides ont été effectués à l'aide d'une rampe équipée de 6 buses 3 filets Albuz (Berthoud, Belleville-sur-Saône, Rhône) permettant de traiter $2 \mathrm{~m}$ de large. Les solutions ont été neutralisées et décantées avant utilisation.

L'azote minéral a été dosé dans chaque parcelle expérimentale sur un échantillon moyen de sol constitué du mélange de 4 carottes ( 2 sur le rang et 2 entre rangs) pour les horizons $0-10$ et $10-20 \mathrm{~cm}$, et de 2 carottes ( 1 sur le rang et 1 entre rangs) pour les hori- 
Tableau II. Principales caractéristiques des essais au champ (essais blocs à 4 répétitions).

\begin{tabular}{lcc}
\hline & Blé & Maïs \\
\hline & & \\
Sol $(\mathrm{pH}$ à l'apport) & $3(6,9)$ & $4(7,3)$ \\
Date de semis & 25 octobre 1986 & 25 avril 1987 \\
Variété & Festival & DEA \\
Parcelle élémentaire $\left(\mathrm{m}^{2}\right)$ & $(2 \times 20)$ & $(3,2 \times 15)$ \\
Date d'apport & 06 avril 1987 & 29 avril 1987 \\
Date de la récolte & 03 septembre 1987 & 22 septembre 1987 \\
Surface récoltée $\left(\mathrm{m}^{2}\right)$ & $(1,25 \times 8)$ & $(1,6 \times 5)$ \\
& &
\end{tabular}

Tableau III. Quantités d'azote et de potassium apportées par les différents traitements appliqués au champ sur blé et maïs.

\begin{tabular}{|c|c|c|c|}
\hline$N\left(\mathrm{~kg} \cdot \mathrm{ha} \mathrm{a}^{-1}\right)$ & $K$ & $p H$ & Volume épandu \\
\hline Total $\quad \mathrm{NNO}_{2}$ & $\left(\mathrm{~kg} h \mathrm{a}^{-1}\right)$ & & $\left(I . h a^{-1}\right)$ \\
\hline
\end{tabular}

Blé :

Témoin ammonitrate

Déchet A 100 liquide

$100 \quad 0$

Déchet A 200 liquide

92

92
192

Déchet $B^{\star} 100$ solide

Déchet $B^{*} 100$ liquide

Maïs :

Témoin ammonitrate

Déchet $B^{\star} 190$ solide

180

190

Déchet $B^{\star} 317$ solide

9

4

131

119

245

140

119

244

296

490

$\begin{array}{cr}- & - \\ 7,8 & 994 \\ 7,8 & 2080 \\ - & - \\ 9,0 & 1687 \\ - & - \\ - & - \\ - & -\end{array}$

* Déchet $\mathrm{B}$ blé : lot 2 tableau $\mathrm{V}$ - maïs : lot 3 tableau $\mathrm{V}$.

zons $20-40$ et $40-60 \mathrm{~cm}$, à chaque prélèvement. L'extraction et le dosage de l'azote nitreux et nitrique étaient effectués le lendemain du jour de prélèvement, selon les méthodes décrites précédemment, sur les échantillons conservés à $+4{ }^{\circ} \mathrm{C}$.

À la récolte (mécanique pour le blé, manuelle pour le maïs) les rendements et teneurs en azote et baryum ont été déterminés sur le grain récolté. Baryum et sodium ont été dosés sur un échantillon moyen du sol des parcelles témoins et des traitements déchet B100 solide (blé) et B317 (maïs).

\section{Essai en serre}

Un essai sur maïs a été mis en place en serre pour comparer les effets de différents modes d'apport du déchet $\mathrm{B}$ (lot 3 , tableau V), à 2 dates différentes : présemis et post-semis. Le déchet a été apporté sous 3 formes en plus du témoin : déchet en poudre, en solution décantée, en solution décantée et neutralisée, témoin $\left(\mathrm{NH}_{4} \mathrm{NO}_{3}+\mathrm{KCl}\right.$ en solution); chaque traitement apportait l'équivalent de $80 \mathrm{mg}$ d'azote et $120 \mathrm{mg}$ de potassium par $\mathrm{kg}$ de sol sec.
Chaque pot était garni d'une couche de billes d'argile expansée (drainage) et de l'équivalent de $4 \mathrm{~kg}$ de sol sec tamisé et homogénéisé (sol 5). L'humidité a été portée à une valeur proche de la capacité au champ par humectation suivie d'un ressuyage de $24 \mathrm{~h}$; cette humidité a été maintenue tout au long de l'essai par arrosage journalier à l'eau.

L'apport de déchet en pré-semis a été réalisé un jour après humectation du sol sur la moitié des pots de l'essai. Une semaine après, 8 graines de maïs (cv.DEA) ont été semées. Un jour après semis, l'apport en post-semis a été réalisé sur la deuxième moitié des pots. La récolte des parties aériennes a été effectuée $35 \mathrm{j}$ après semis. Le nombre de plantes et la matière sèche ont été déterminés sur chaque pot.

\section{Interprétation des résultats}

Quand les effets traitements sont statistiquement significatifs lors de l'analyse de variance, les moyennes ont été comparées par un test Newman et Keuls. Dans les tableaux, les valeurs suivies de la (des) même(s) lettres(s) ne diffèrent pas significativement 
au seuil $P=0,05$. Les coefficients de variation précisent la dispersion des résultats.

\section{RÉSULTATS ET DISCUSSION}

\section{Composition des déchets}

La somme des éléments du tableau IV nous montre que les sels caloporteurs (déchet $A$ ) ne sont pratiquement constitués que de nitrites et de nitrates de potassium et de sodium, totalement solubles ( $\mathrm{pH}$ de la solution de $35 \mathrm{~g}$ dans $100 \mathrm{~cm}^{3}$ : $12,7)$. Les autres éléments sont en très faible quantité et ne posent pas de problèmes de toxicité.

Les sels de bain de trempe (déchet $B$ ), initialement composés de nitrites et de nitrates de potassium et de sodium sont contaminés par les bains de traitement précédents (cémentation, etc). Une partie des composants n'est pas soluble $\left(\mathrm{pH}\right.$ de la solution de $35 \mathrm{~g}$ dans $100 \mathrm{~cm}^{3}$ : entre 11 et 11,5 selon le lot). On retrouve essentiellement nitrites, nitrates, chlorures et carbonates de sodium, potassium et baryum (tableau V); Cu (3-4 mg. $\mathrm{kg}^{-1}$ ), Mn (45-70 mg.kg-1), Zn (5-22 mg. kg ${ }^{-1}$ ) et Co (2-20 mg. $\left.\mathrm{kg}^{-1}\right)$, non reportés dans le tableau $V$, sont également présents. Leur composition chimique varie légèrement

Tableau IV. Composition des sels caloporteurs (déchet A).

\begin{tabular}{lcc}
\hline Élément & $\begin{array}{c}\text { Quantité } \\
\text { totale }\end{array}$ & $\begin{array}{c}\text { Fraction } \\
\text { soluble }\end{array}$ \\
\hline$\left(\mathrm{g} .100 \mathrm{~g} \mathrm{~g}^{-1} \mathrm{sec}\right)$ & & \\
$\mathrm{N}_{\mathrm{NO} 3}$ & 7,9 & 7,9 \\
$\mathrm{~N}_{\mathrm{NO} 2}$ & 9,0 & 9,0 \\
$\mathrm{~K}$ & 21,5 & 21,7 \\
$\mathrm{Na}$ & 15,4 & 15,3 \\
$\mathrm{Ba}$ & 0,035 & $<0,001$ \\
& & \\
$\left(\mathrm{mg} \cdot \mathrm{kg}^{-1} \mathrm{sec}\right)$ & & 3,1 \\
$\mathrm{Cr}$ & 4,6 & $<10,0^{*}$ \\
$\mathrm{Ni}$ & 5,9 & 1,7 \\
$\mathrm{Li}$ & 1,9 & $5,9^{*}$ \\
$\mathrm{~Pb}$ & 3,3 & $<0,1$ \\
$\mathrm{Cd}$ & $<0,1$ & \\
$\mathrm{Sr}$ & $<5,0$ & \\
\hline
\end{tabular}

- : non déterminé; * : la sensibilité relative sur ces déterminations explique les différences total-soluble. d'une chaîne de fabrication à l'autre ( 5 chaînes ont été suivies en juillet-août 1987). Dans le temps d'importantes variations des teneurs en baryum, lithium, chrome et nickel sont observées. Ces variations traduisent vraisemblablement une variation des apports des bains précédents en fonction de la forme et de la taille des objets traités. Les cyanures, utilisés lors de la cémentation, se trouvent en quantités non négligeables : de l'ordre de 300 g.t $\mathrm{t}^{-1}$.

La forte alcalinité de ces produits nous conduit à envisager leur utilisation après mise en solution dans l'eau et neutralisation. La neutralisation de solutions à $350 \mathrm{~g} . \mathrm{I}^{-1}$ nécessite 20 méq $\mathrm{H}^{+}$pour $100 \mathrm{~g}$ de déchet $\mathrm{A}$, de 30 à 60 méq $\mathrm{H}^{+}$pour $100 \mathrm{~g}$ de déchet $\mathrm{B}$, pour abaisser le $\mathrm{pH}$ aux environs de 7,5 (fig 1). La mise en solution du déchet $B$ entraîne une élimination importante d'éléments indésirables par précipitation (tableau V, lot 2); l'acide sulfurique complète l'élimination du baryum mais solubilise le chrome, à l'inverse de l'acide chlorhydrique (tableau VI). Lors de cette neutralisation, des précautions sont à prendre, les cyanures pouvant se transformer en acide cyanhydrique, volatile dès que le $\mathrm{pH}$ descend en-dessous de 9 (Charlot, 1966).

Les déchets étudiés contiennent des éléments fertilisants : $170 \mathrm{~kg}$ de $\mathrm{N}$ et $215 \mathrm{~kg}$ de K.t-1 pour le déchet $A$, et, en moyenne, $100 \mathrm{~kg}$ de $\mathrm{N}$ et $154 \mathrm{~kg}$ de $\mathrm{K}$ pour le déchet $\mathrm{B}$. Les autres éléments sont en faible quantité, Ba excepté, mais les éléments-traces contenus dans les sels de bain de trempe doivent cependant être pris en considération. En appliquant la norme NF U 44041 (boues des ouvrages d'épuration des eaux

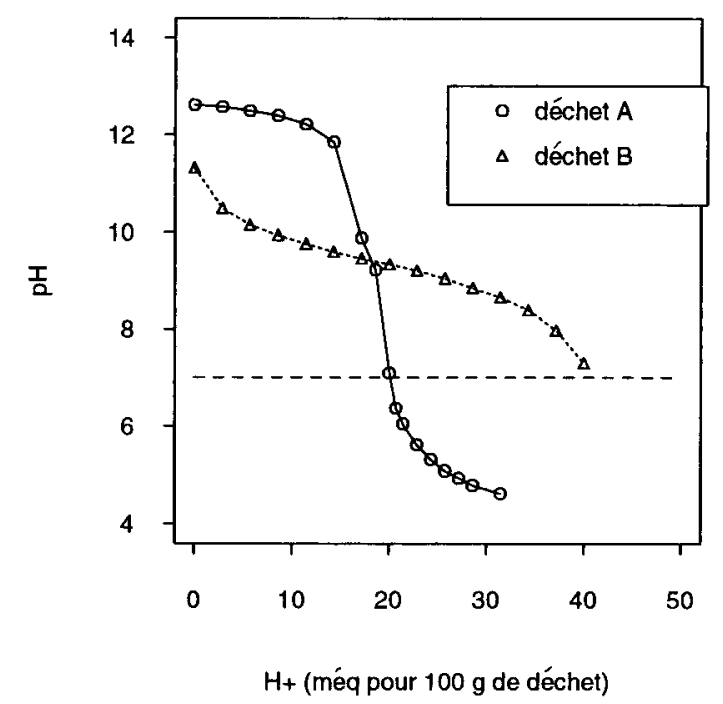

Fig 1. Courbes de neutralisation des déchets $A$ et $B$ (solution de $35 \mathrm{~g}$ de déchet dans $100 \mathrm{~cm}^{3}$ d'eau, neutralisation par $\mathrm{HCl} 0,1 \mathrm{~N}$ ). 
Tableau V. Composition moyenne des sels de bain de trempe (déchet B) à différentes dates de prélèvement.

\begin{tabular}{|c|c|c|c|c|c|}
\hline \multirow[t]{2}{*}{ Élément } & \multirow{2}{*}{$\begin{array}{c}1981 \\
\text { lot } 1 \\
\text { Total }\end{array}$} & \multicolumn{2}{|c|}{$\begin{array}{c}\text { Mars } 1987 \\
\quad \text { lot } 2\end{array}$} & \multirow{2}{*}{$\begin{array}{c}\text { Avril } 1987 \\
\text { lot } 3 \\
\text { Total }\end{array}$} & \multirow{2}{*}{$\begin{array}{c}\text { Juil-août } 1987^{*} \\
\text { Total }\end{array}$} \\
\hline & & Total & Soluble & & \\
\hline \multicolumn{6}{|c|}{ (g.100 $\left.\mathrm{g}^{-1} \mathrm{MS}\right)$} \\
\hline $\mathrm{N}_{\mathrm{NO} 3}$ & 4,2 & 5,3 & 5,3 & 5,1 & $4,3-5,5$ \\
\hline $\mathrm{N}_{\mathrm{NO} 2}$ & 5,1 & 5,3 & 5,3 & 6,1 & $4,7-6,0$ \\
\hline $\mathrm{K}$ & 13,9 & 14,8 & 14,1 & 17,3 & $14,1-16,9$ \\
\hline $\mathrm{Na}$ & 15,0 & 16,3 & 15,2 & 15,0 & $15,8-16,2$ \\
\hline $\mathrm{Ba}$ & 17,1 & 11,7 & 0,02 & 6,8 & $9,5-13,1$ \\
\hline $\mathrm{Cl}$ & 9,7 & - & - & - & $6,6-10,1$ \\
\hline $\mathrm{CO}_{3}$ & 14,2 & - & - & - & $6,3-8,8$ \\
\hline \multicolumn{6}{|c|}{ (mg.kg-1 MS) } \\
\hline $\mathrm{Cr}$ & 5 & 339 & 44 & 268 & $226-316$ \\
\hline $\mathrm{Ni}$ & 17 & 269 & $<10$ & 147 & $127-410$ \\
\hline $\mathrm{Li}$ & 928 & 8,5 & 1,7 & 4,8 & $3,9-5,2$ \\
\hline $\mathrm{Pb}$ & 19 & 3,2 & $4,1^{*}$ & 13,4 & $8,9-40,5$ \\
\hline $\mathrm{Cd}$ & 2,8 & $<0,1$ & $<0,1$ & 0,19 & $<0,5$ \\
\hline $\mathrm{Sr}$ & 136 & 293 & - & 115 & $120-200$ \\
\hline $\mathrm{CN}$ & - & - & - & - & $100-700$ \\
\hline
\end{tabular}

* voir tableau IV; ** valeurs extrêmes sur 5 chaînes de fabrication; - : non déterminé.

Tableau VI. Influence de la nature de l'acide de neutralisation sur la teneur en divers éléments minéraux du surnageant (déchet B).

mg. $\mathrm{kg}^{-1}$ du produit sec de départ

$\begin{array}{llll}\text { Chrome Nickel } & \text { Baryum } & \text { Lithium } & \text { Strontium }\end{array}$

\begin{tabular}{lrrrrr} 
Teneur initiale & 285 & 230 & 95000 & 4 & 120 \\
Neutralisation $\mathrm{HCl}$ & 40 & 2 & 465 & 1 & $<3$ \\
Neutralisation $\mathrm{H}_{2} \mathrm{SO}_{4}$ & 267 & 2 & 5 & 1 & $<3$ \\
\hline
\end{tabular}

urbaines) aux valeurs maximales du tableau $\mathrm{V}$, seuł $\mathrm{Ni}$ pose problème et limiterait la dose annuelle de déchet solide à 1,5 t.ha-1; toutefois la mise en solution élimine la presque totalité de cet élément, ainsi que $\mathrm{Ba}$.

\section{Transformation de l'azote nitreux dans le sol}

\section{Mesures de laboratoire}

\section{Première série d'incubations}

L'évolution des nitrites apportés par le déchet $B$ et leur incidence sur la dynamique de l'azote minéral sont très différentes dans les 2 sols utilisés.
Dans le sol 1, à pH 7,9 (fig 2), la nitrification se fait très rapidement : $60 \mathrm{mg}$ de l'azote ammoniacal sont transformés en azote nitrique en moins d'une semaine sans accumulation d'azote nitreux. L'azote nitreux apporté par le déchet ou par le nitrite de potassium est transformé en azote nitrique, à une vitesse d'au moins $60 \mathrm{mg}$ par semaine jusqu'à la dose $60 \mathrm{mg} \cdot \mathrm{kg}^{-1}$, et moins rapidement lors de la première semaine (40-45 mg) pour les doses supérieures qui rattrapent lors de la seconde semaine $(75 \mathrm{mg})$. Le comportement du sel de trempe à la dose $60 \mathrm{mg}$ est comparable à celui du nitrite de potassium. On observe un léger effet azote sur la respiration du sol, qui n'est stimulée que pendant la première semaine. 

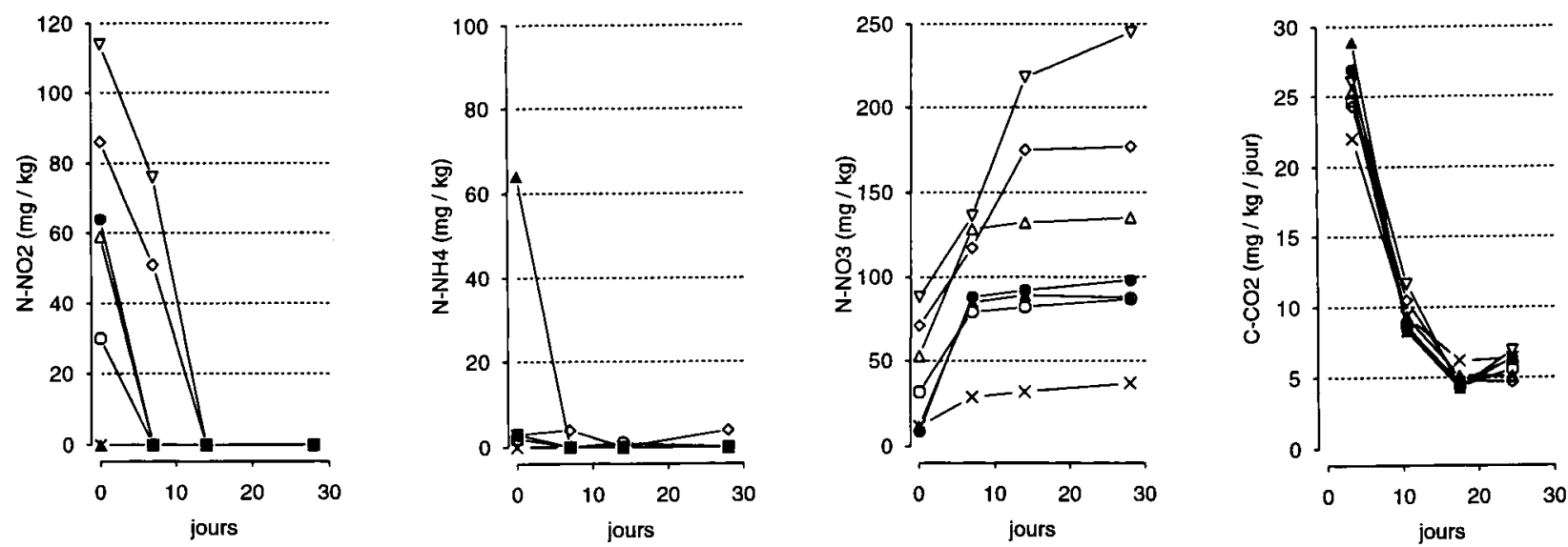

Fig 2. Évolution de l'azote minéral et production de $\mathrm{C}_{\mathrm{CO}_{2}}$ dans le sol 1 ayant reçu différentes doses de déchet $\mathrm{B}$ (témoins : $\mathrm{x}$ non traité, nitrite $60 \mathrm{mgN}, \Delta$ ammonium $60 \mathrm{mgN}$ - déchet $\mathrm{B}: 030 \mathrm{mg} \mathrm{N}_{\mathrm{NO}_{2}}, \Delta 60 \mathrm{mg} \mathrm{N} \mathrm{NO}_{2}, \diamond 90 \mathrm{mg} \mathrm{N}_{\mathrm{NO}_{2}}, \nabla 120 \mathrm{mg} \mathrm{N} \mathrm{NO}_{2}$ ).

Dans le sol 2, à pH 4,6 (fig 3), la nitrification de l'ammonium ne se fait que très lentement : la production d'azote nitrique n'est pas plus importante que celle du témoin. La disparition de l'azote nitreux se fait en 2 temps. Dans l'heure qui suit l'apport, une disparition quasi instantanée de $80 \%$ de la quantité apportée est observée (tableau VII), avec corrélativement une apparition d'azote nitrique (de 40 à $60 \%$ de l'azote nitreux disparu) sans production détectable de protoxyde d'azote. On est vraisemblablement en présence d'un phénomène de décomposition de l'acide nitreux selon une réaction de dismutation qui pourrait être équilibrée selon l'équation :

$$
5 \mathrm{HNO}_{2} \longrightarrow 3 \mathrm{HNO}_{3}+\mathrm{N}_{2}+\mathrm{H}_{2} \mathrm{O}
$$

Cette décomposition de l'acide nitreux en sol acide a été rapportée par différents auteurs et
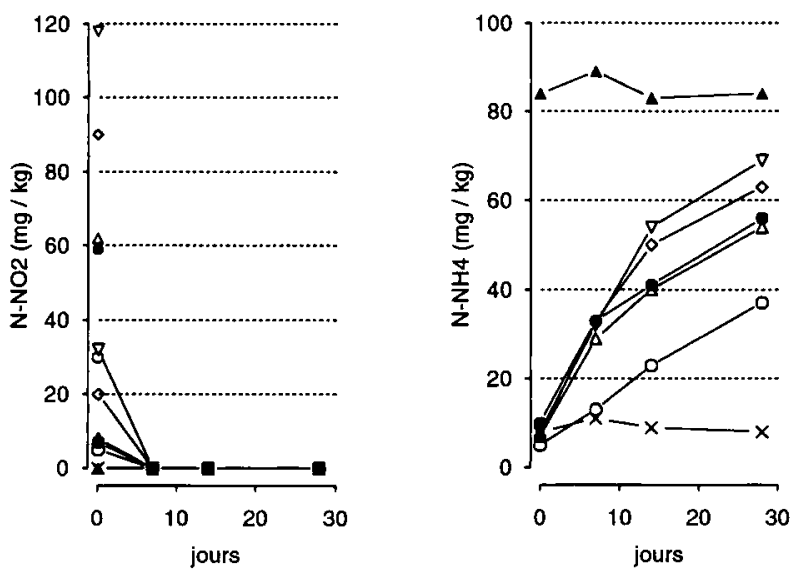

plus particulièrement par Nelson et Bremmer (1970). La production d'acide nitrique en milieu aéré peut également découler de l'oxydation de NO, l'un des produits de la décomposition de $\mathrm{H}_{2} \mathrm{NO}_{3}$, en $\mathrm{NO}_{2}$ qui au contact de l'eau se transforme en acide nitrique (Pascal, 1956).

Dans la semaine qui suit, les nitrites restants disparaissent complètement sans production simultanée d'azote nitrique (fig 3). On peut penser à plusieurs voies possibles pour expliquer cette disparition : une intégration des nitrites au turnover de l'azote, et parallèlement une augmentation de la minéralisation en $\mathrm{NH}_{4}^{+}$et une augmentation de la respiration; ou des réactions de désamination nitreuse de type Van Slike conduisant à la production de $\mathrm{N}_{2}$ (Gerretsen et de Hoop, 1957; Christianson et al, 1979). La forte augmentation de l'activité respiratoire et de la production d'ammonium (tableau VII) lorsque la
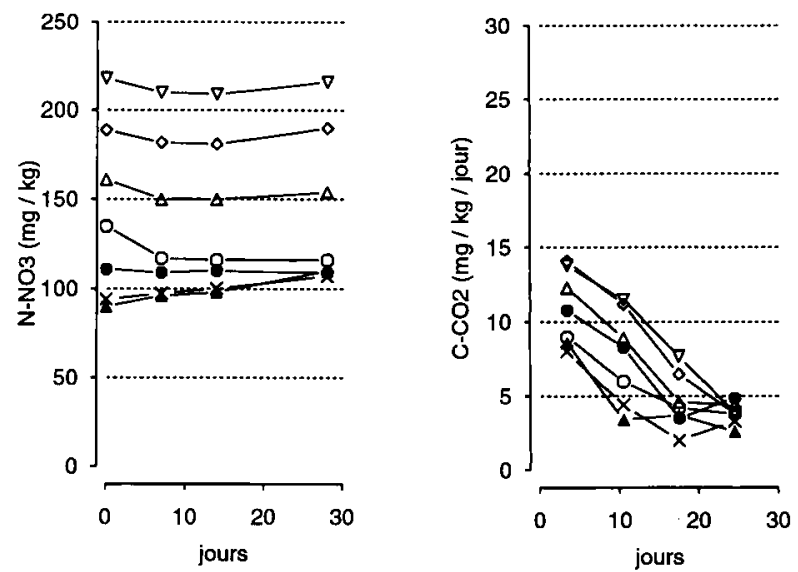

Fig 3. Evolution de l'azote minéral et production de $\mathrm{C}_{\mathrm{CO}_{2}}$ dans le sol 2 ayant reçu différentes doses de déchet $\mathrm{B}$ (légende : se reporter à la figure 2). Les points isolés représentent les valeurs théoriques à $t_{0}$. 
Tableau VII. Évolution de l'azote et du carbone dans le sol 2 après apport d'ions nitreux (moyenne de 4 répétitions, valeurs exprimées en $\mathrm{mg} \mathrm{kg}^{-1}$ de $\mathrm{C}$ ou de $\mathrm{N}$ ).

Première heure d'incubation

\begin{tabular}{|c|c|c|}
\hline $\begin{array}{l}\mathrm{N}_{\mathrm{NO}_{2} \text { apporté }} \\
\left(m g . \mathrm{kg}^{-1}\right)\end{array}$ & $\begin{array}{l}\mathrm{N}_{\mathrm{NO}_{2}}(1) \\
\text { disparu }\end{array}$ & $\begin{array}{l}\mathrm{N}_{\mathrm{NO}_{2}} \text { (2) } \\
\text { formé }\end{array}$ \\
\hline
\end{tabular}

Après 4 semaines

\begin{tabular}{cccccc}
\hline 0 (témoin) & - & - & - & 138 & 0 \\
30 (déchet $\mathrm{B})$ & 23 & 9 & 0,39 & 168 & 29 \\
60 (déchet $\mathrm{B})$ & 48 & 26 & 0,54 & 210 & 46 \\
90 (déchet B) & 64 & 37 & 0,58 & 248 & 55 \\
120 (déchet B) & 80 & 37 & 0,46 & 196 & 61 \\
$60\left(\mathrm{KNO}_{2}\right)$ & - & - & - & 128 & 48 \\
$\left.60\left(\mathrm{NH}_{4}\right)_{2} \mathrm{SO}_{4}\right)$ & - & - & - & & 0 \\
\hline
\end{tabular}

dose de nitrites augmente est vraisemblablement la conséquence d'un effet toxique en milieu acide de l'azote nitreux, et éventuellement des éléments d'accompagnement, sur une partie de la microflore du sol qui est tuée et sert de substrat aux microorganismes résistants, d'où cette minéralisation (Chaussod et al, 1986b).

La disparition des ions nitrites dans ces 2 sols est assez rapide, que les mécanismes de nitrification soient efficaces (sol 1) ou non (sol 2). Le devenir de l'azote nitreux du déchet ou du nitrite de potassium dépend fortement du $\mathrm{pH}$ qui conditionne sa transformation biologique. En sol neutre il se retrouve intégralement sous forme nitrique; en sol acide une partie importante est perdue par des mécanismes de dénitrification chimique, phénomène que certains auteurs signalent aussi pour des $\mathrm{pH}$ supérieurs à 6 (Nelson et Bremner, 1969; Christianson et al, 1979).

\section{Deuxième série d'incubations}

L'effet de la température sur la vitesse d'oxydation des nitrites apparaît très nettement dans cet essai (fig 4) : $52 \mathrm{mg}$ d'azote nitreux sont oxydés en une semaine à $20^{\circ} \mathrm{C}, 38 \mathrm{mg}$ à $15^{\circ} \mathrm{C}$ et 22 mg à $10^{\circ} \mathrm{C}$, le $Q_{10}$ (calculé entre 10 et $20^{\circ} \mathrm{C}$ ) ayant une valeur de 2,2 . Vers $2{ }^{\circ} \mathrm{C}$ l'activité de Nitrobacter, qui assure le passage nitrite-nitrate, cesse (Justice et Smith, 1962).

La période d'épandage, en déterminant la température du sol, permet de prévoir la rapidité de disparition de l'azote nitreux : dès que les températures avoisinent $10^{\circ} \mathrm{C}$, un apport de 20 mg.kg-1 (environ $50 \mathrm{~kg}^{-1} \mathrm{ha}^{-1}$ ) devrait être oxydé en moins d'une semaine.

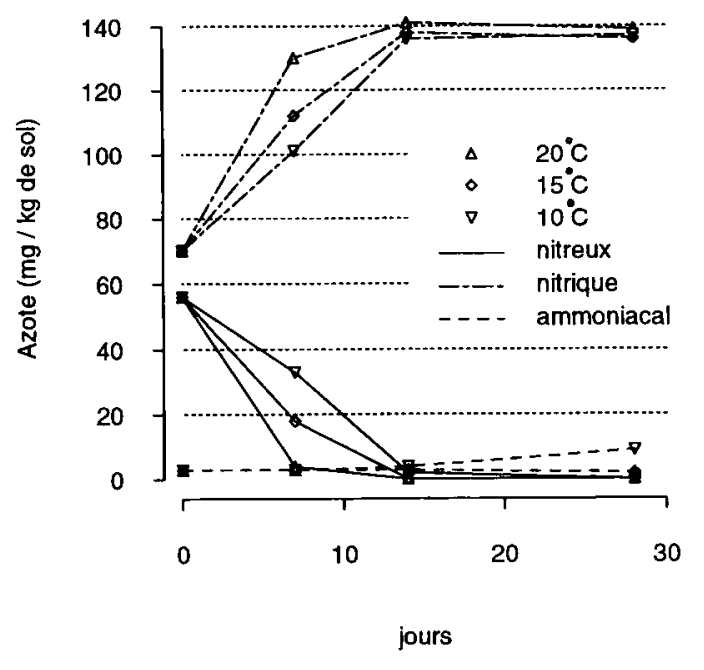

Fig 4. Effet de la température sur la transformation des nitrites du déchet $B$ en nitrates dans le sol 1.

\section{Mesures au champ}

Sous blé (tableau VIII), l'azote nitreux apporté par les déchets se transforme rapidement dans les conditions de cet essai : $4 \mathrm{j}$ après apport des produits on retrouve dans le sol moins du quart de l'apport et la disparition est complète après 15 $\mathrm{j}$, la transformation apparaissant la plus lente dans le traitement déchet B 100 solide; au cours de cette période la température moyenne du sol à $-5 \mathrm{~cm}$ a été de $8,6^{\circ} \mathrm{C}$. Pour des apports d'azote minéral de même ordre, les teneurs en azote nitrique du sol des traitements liquides $A$ 100 et $B 100$ sont analogues à celles du témoin ammonitrate; l'apport B 100 solide se traduit par contre par une forte élévation du niveau d'azote 
Tableau VIII. Évolution de la teneur du sol en azote nitrique et nitreux pendant la végétation du blé (moyenne de 4 répétitions).

\begin{tabular}{|c|c|c|c|c|c|c|c|c|}
\hline \multirow[b]{2}{*}{$\begin{array}{l}\text { Dates } \\
\text { jours } \\
\text { après apport }\end{array}$} & \multicolumn{4}{|c|}{$N_{N O 3}\left(m g \cdot k^{-1}\right)$} & \multicolumn{4}{|c|}{$N_{\mathrm{NO} 2}\left(m g \cdot \mathrm{kg}^{-1}\right)$} \\
\hline & $\begin{array}{c}10 \text { avril } \\
4\end{array}$ & $\begin{array}{c}14 \text { avril } \\
8\end{array}$ & $\begin{array}{c}21 \text { avril } \\
15\end{array}$ & $\begin{array}{c}03 \text { août } \\
R^{\star}\end{array}$ & $\begin{array}{c}10 \text { avril } \\
4\end{array}$ & $\begin{array}{c}14 \text { avril } \\
8\end{array}$ & $\begin{array}{c}21 \text { avril } \\
15\end{array}$ & $\begin{array}{c}03 \text { août } \\
R\end{array}$ \\
\hline \multicolumn{9}{|l|}{$0.10 \mathrm{~cm}$} \\
\hline témoin & 26,8 & $26,2^{b}$ & $19,4^{a b}$ & $4,1^{b}$ & 0 & $0^{b}$ & 0 & 0 \\
\hline A 100 & 33,0 & $38,7^{a b}$ & $13,7^{b}$ & $3,9^{b}$ & 0,9 & $0,4^{b}$ & 0 & 0 \\
\hline A 200 & 55,4 & $58,7^{a}$ & $46,9^{a}$ & $6,9^{a}$ & 8,6 & $4,1^{a b}$ & 0 & 0 \\
\hline B $100 \mathrm{sol}$ & 37,1 & $65,4^{a}$ & $33,1^{\mathrm{ab}}$ & $4,8^{b}$ & 9,3 & $7,1^{\mathrm{a}}$ & 0 & 0 \\
\hline B 100 liq & 29,5 & $17,5^{\mathrm{b}}$ & $28,5^{\mathrm{ab}}$ & $4,3^{b}$ & 1,6 & $0^{b}$ & 0 & 0 \\
\hline $\mathrm{CV}$ & $39 \%$ & $27 \%$ & $41 \%$ & $24 \%$ & $123 \%$ & $117 \%$ & & \\
\hline \multicolumn{9}{|l|}{$10-20 \mathrm{~cm}$} \\
\hline témoin & 13,8 & $7,4^{\mathrm{C}}$ & 10,3 & $3,4^{b}$ & 0 & 0 & 0 & 0 \\
\hline A 100 & 7,4 & $7,3^{c}$ & 9,7 & $3,8^{b}$ & 0 & 0 & 0 & 0 \\
\hline A 200 & 16,2 & $25,5^{\mathrm{a}}$ & 12,4 & $4,9^{a}$ & 0,6 & 0 & 0 & 0 \\
\hline B $100 \mathrm{sol}$ & 22,5 & $18,0^{b}$ & 9,7 & $4,0^{b}$ & 0,9 & 0,6 & 0 & 0 \\
\hline B 100 liq & 8,1 & $6,5^{c}$ & 9,2 & $3,6^{b}$ & 0,2 & 0 & 0 & 0 \\
\hline $\mathrm{CV}$ & $62 \%$ & $36 \%$ & $53 \%$ & $14 \%$ & $250 \%$ & & & \\
\hline \multicolumn{9}{|l|}{$20-40 \mathrm{~cm}$} \\
\hline témoin & $-{ }^{* *}$ & - & $5,3^{a}$ & 2,4 & - & - & 0 & 0 \\
\hline A 100 & - & - & $3,8^{b}$ & 2,1 & - & - & 0 & 0 \\
\hline A 200 & - & - & $3,5^{b}$ & 2,8 & - & - & 0 & 0 \\
\hline B $100 \mathrm{sol}$ & - & - & $2,6^{b}$ & 2,2 & - & - & 0 & 0 \\
\hline B $100 \mathrm{liq}$ & - & - & $3,0^{b}$ & 2,1 & - & - & 0 & 0 \\
\hline $\mathrm{CV}$ & & & $25 \%$ & $27 \%$ & & & & \\
\hline \multicolumn{9}{|l|}{$40-60 \mathrm{~cm}$} \\
\hline témoin & - & - & 1,6 & 1,2 & - & - & 0 & 0 \\
\hline A 100 & - & - & 2,2 & 0,8 & - & - & 0 & 0 \\
\hline A 200 & - & - & 2,0 & 1,3 & - & - & 0 & 0 \\
\hline B $100 \mathrm{sol}$ & - & - & 2,2 & 0,8 & - & - & 0 & 0 \\
\hline B $100 \mathrm{liq}$ & - & - & 2,3 & 0,8 & - & - & 0 & 0 \\
\hline $\mathrm{CV}$ & & & $43 \%$ & $30 \%$ & & & & \\
\hline
\end{tabular}

Les valeurs suivies de la(les) même(s) lettre(s) ne diffèrent pas au seuil $P=0,05$ (Newman et Keuls).

${ }^{*} \mathrm{R}$ : à la récolte; ${ }^{\star \star}-$ : non déterminé.

Avant traitement le 6 avril (moyenne de 20 prélèvements)

\begin{tabular}{|r|c|c|}
\hline & $N_{\mathrm{NO} 3}$ & $N_{\mathrm{NO} 2}$ \\
\hline $0-10 \mathrm{~cm}$ & 6,5 & 0 \\
$10-20 \mathrm{~cm}$ & 4,0 & 0 \\
$20-40 \mathrm{~cm}$ & 2,7 & 0 \\
$40-60 \mathrm{~cm}$ & 1,3 & 0 \\
\hline
\end{tabular}

nitrique au $8^{\mathrm{e}} \mathrm{j}$, dépassant la teneur observée dans le traitement A 200 : cette accumulation temporaire peut traduire une perturbation momentanée de l'assimilation des plantes alors au stade montaison, liée vraisemblablement aux concentrations inhabituelles en ions nitreux au niveau des racines. À la récolte, le faible reliquat d'azote nitrique est plus important pour le traitement déchet A 200.

Sous maïs (tableau IX), l'azote nitreux disparaît du profil en 4 semaines dans le traitement déchet B 190, alors qu'il faut attendre 6 semaines pour ne plus en trouver dans le traitement déchet $B$ 317; la température moyenne du 
Tableau IX. Évolution de la teneur du sol en azote nitrique et nitreux pendant la végétation du maïs (moyenne de 4 répétitions).

\begin{tabular}{|c|c|c|c|c|c|c|c|c|c|c|c|c|}
\hline \multirow[b]{2}{*}{$\begin{array}{l}\text { Date } \\
\text { jours } \\
\text { après } \\
\text { apport }\end{array}$} & \multicolumn{6}{|c|}{$N_{\text {NO3 }}\left(m g \cdot k g^{-1}\right)$} & \multicolumn{6}{|c|}{$N_{\mathrm{NO} 2}\left(m g \cdot \mathrm{kg}^{-1}\right)$} \\
\hline & $\begin{array}{c}6 \text { mai } \\
7\end{array}$ & $\begin{array}{c}12 m a i \\
13\end{array}$ & $\begin{array}{c}20 \mathrm{mai} \\
21\end{array}$ & $\begin{array}{c}26 \text { mai } \\
27\end{array}$ & $\begin{array}{c}12 \text { juin } \\
44\end{array}$ & $\begin{array}{c}22 \text { sept } \\
R^{*}\end{array}$ & $\begin{array}{c}6 \text { mai } \\
7\end{array}$ & $\begin{array}{c}12 \text { mai } \\
13\end{array}$ & $\begin{array}{c}20 \text { mai } \\
21\end{array}$ & $\begin{array}{c}26 \text { mai } \\
27\end{array}$ & $\begin{array}{l}12 \text { juin } \\
44\end{array}$ & $\begin{array}{c}22 \text { sept } \\
R^{*}\end{array}$ \\
\hline $0-10 \mathrm{~cm}$ & & & & & & & & & & & & \\
\hline témoin & 32,6 & 58,6 & 39,0 & 37,9 & - & 2,4 & 0,3 & 2,0 & 0,8 & 0 & - & 0 \\
\hline B 190 & 48,2 & 53,5 & 21,5 & 25,0 & - & 2,5 & 21,6 & 11,1 & 1,6 & 0 & - & 0 \\
\hline B 317 & 70,4 & 93,4 & 36,5 & 46,3 & 32,1 & 2,7 & 42,2 & 62,9 & 7,0 & 6,1 & 0 & 0 \\
\hline CV & $46 \%$ & $43 \%$ & $43 \%$ & $55 \%$ & & $26 \%$ & $93 \%$ & $114 \%$ & $190 \%$ & & & \\
\hline $10-20 \mathrm{~cm}$ & & & & & & & & & & & & \\
\hline $\begin{array}{l}\text { témoin } \\
\text { B } 190\end{array}$ & $\begin{array}{l}35,3 \\
46,8\end{array}$ & $\begin{array}{l}41,5 \\
40,9\end{array}$ & $\begin{array}{l}37,1 \\
44,6\end{array}$ & $\begin{array}{l}26,9 \\
22,9\end{array}$ & $\begin{array}{l}- \\
-\end{array}$ & $\begin{array}{l}2,9 \\
2,7\end{array}$ & $\begin{array}{c}0 \\
26,2\end{array}$ & $\begin{array}{l}1,2^{\mathrm{b}} \\
8,6^{\mathrm{b}}\end{array}$ & $\begin{array}{r}0,8 \\
11,1\end{array}$ & $\begin{array}{l}0 \\
0\end{array}$ & $\begin{array}{l}- \\
-\end{array}$ & $\begin{array}{l}0 \\
0\end{array}$ \\
\hline B 317 & 40,3 & 61,7 & 44,4 & 41,8 & 61,4 & 3,4 & 24,8 & $22,4^{a}$ & 6,0 & 2,1 & 0 & 0 \\
\hline $\mathrm{CV}$ & $61 \%$ & $36 \%$ & $70 \%$ & $35 \%$ & & $13 \%$ & $133 \%$ & $66 \%$ & $247 \%$ & & & \\
\hline $20-40 \mathrm{~cm}$ & & & & & & & & & & & & \\
\hline témoin & 18,7 & 20,1 & 30,1 & 8,9 & - & $2,4^{b}$ & 0,2 & 0,8 & $0,7^{b}$ & 0 & - & 0,2 \\
\hline B 190 & 24,2 & 13,1 & 20,5 & 18,8 & - & $2,3^{b}$ & 8,5 & 1,4 & $0^{\mathrm{b}}$ & 0 & - & 0,2 \\
\hline B 317 & 16,4 & 15,1 & 45,1 & 30,4 & - & $4,1^{a}$ & 5,4 & 1,5 & $14,3^{a}$ & 0,3 & - & 0,3 \\
\hline CV & $53 \%$ & $55 \%$ & $59 \%$ & $85 \%$ & & $22 \%$ & $160 \%$ & $138 \%$ & $130 \%$ & & & $32 \%$ \\
\hline $40-60 \mathrm{~cm}$ & & & & & & & & & & & & \\
\hline témoin & 6,7 & 7,7 & 14,0 & 9,5 & - & $1,6^{b}$ & 0 & 0 & 0 & 0 & - & 0,3 \\
\hline B 190 & 8,0 & 7,9 & 10,0 & 14,9 & - & $1,6^{b}$ & 1,8 & 1,4 & 0 & 0,4 & - & 0,2 \\
\hline B 317 & 7,8 & 8,0 & 12,7 & 8,0 & - & $9,9 a$ & 2,1 & 1,3 & 4,3 & 0 & - & 0,2 \\
\hline $\mathrm{CV}$ & $32 \%$ & $44 \%$ & $65 \%$ & $60 \%$ & & $47 \%$ & $101 \%$ & $153 \%$ & $166 \%$ & & & $87 \%$ \\
\hline
\end{tabular}

Les valeurs suivies de la(les) même(s) lettre(s) ne diffèrent pas au seuil $P=0,05$ (Newman et Keuls).

$R$ : à la récolte; - : non déterminé.

Avant traitement le 29 avril (moyenne de 10 prélèvements)

\begin{tabular}{|r|l|l|}
\hline & $N_{\mathrm{NO} 3}$ & $\mathrm{~N}_{\mathrm{NO} 2}$ \\
\hline $0-10 \mathrm{~cm}$ & 8,6 & 0,1 \\
$10-20 \mathrm{~cm}$ & 8,4 & 0 \\
$20-40 \mathrm{~cm}$ & 6,6 & 0 \\
$40-60 \mathrm{~cm}$ & 5,5 & 0 \\
\hline
\end{tabular}

sol à $-5 \mathrm{~cm}$ a été de $11,5^{\circ} \mathrm{C}$ pendant cette période. Les valeurs observées dans les horizons profonds indiquent qu'il y a eu lessivage, mais de façon hétérogène sur l'essai (forts coefficients de variation). Les teneurs en azote nitrique mesurées sous le traitement B 190 sont comparables à celles du traitement témoin; la dose $B$ 317 provoque une augmentation normale de ces teneurs, compte-tenu de la quantité d'azote minéral apportée plus importante. À la récolte, le reliquat d'azote nitrique du traitement déchet $B$ 317 est supérieur à celui des autres traitements, à partir de l'horizon $10-20 \mathrm{~cm}$ et surtout dans I'horizon 40-60 cm.
Les ions nitreux apportés sur le blé en début de montaison disparaissent en 1-2 semaines. En sol nu (semis de maïs) il leur faut 4-6 semaines. Outre le fait que les sols sont différents, d'autres facteurs pourraient expliquer cette persistance plus ou moins longue:

- la quantité de nitrite apportée (déchet $A$ aux doses 100 et 200 sur blé, déchet $B$ aux doses 190 et 317 sur maïs). La persistance augmente dans la proportion des apports, comme cela avait été observé en incubation de laboratoire dans le sol 1.

- la forme d'apport. La vitesse de disparition de l'azote nitreux correspond à peu près à ce que 
l'expérience de laboratoire permettait de prévoir lorsque les déchets sont apportés sous forme liquide (42 à $47 \mathrm{~kg} \cdot \mathrm{ha}^{-1}$.semaine ${ }^{-1}$ ) alors que la température du sol, durant la période de présence des nitrites se situe autour de $10^{\circ} \mathrm{C}$ (blé : 8,6 ; maïs : 11,5). II n'en est pas de même lors des apports sous forme solide sur blé et maïs, la vitesse diminuant environ de moitié $\left(25 \mathrm{~kg} \mathrm{ha}^{-1}\right.$. semaine-1).

Lors d'un apport du déchet B sous forme solide, la transformation de l'azote nitreux est ralentie. Ce phénomène peut correspondre à un dysfonctionnement de la microflore, déjà observé lors des incubations de laboratoire, et avoir plusieurs origines : la forte accumulation d'ions nitreux autour des particules épandues dont la dissolution est lente, une élévation du $\mathrm{pH}$ liée à l'alcalinité de ces particules, ou la présence d'éléments toxiques, non éliminés comme lors de la mise en solution-neutralisation-décantation. L'apport sous forme solide d'une forte dose de sels de trempe se traduit alors par un lessivage de l'azote nitreux jusqu'à $60 \mathrm{~cm}$, que l'on retrouve sous forme nitrique à la récolte (maïs).

\section{Suivi des autres éléments}

Sur blé (tableau X) le déchet B apportant 110 $\mathrm{kg} \cdot \mathrm{ha}^{-1}$ de baryum provoque un enrichissement de $18 \%$ de l'horizon $0-10 \mathrm{~cm}$. Sur maïs (tableau XI) l'apport étant plus important (193 kg.ha-1) l'augmentation des teneurs est plus forte et se manifeste jusqu'à $40 \mathrm{~cm}$.

Tableau $\mathbf{X}$. Teneur du sol en différents éléments minéraux et $\mathrm{pH}$ à la récolte du blé.

$\begin{array}{ccc}\text { Baryum } & \text { Sodium } & \mathrm{pH} \\ \text { total } & \text { échangeable } & \\ \left(\mathrm{mg}_{\mathrm{kg}} \mathrm{kg}^{-1}\right) & \left(\text { méq. } 100 \mathrm{~g}^{-1}\right)\end{array}$

\begin{tabular}{llll}
$\begin{array}{l}\text { 0-10 cm } \\
\text { Témoin }\end{array}$ & 283 & 0,058 & 6,6 \\
$\begin{array}{l}\text { Déchet B solide } \\
\text { 10-20 cm }\end{array}$ & 333 & 0,247 & 6,7 \\
$\quad$ Témoin & 277 & 0,074 & 6,4 \\
$\quad$ Déchet B solide & 284 & 0,166 & 6,8 \\
$\begin{array}{l}\text { 20-40 cm } \\
\text { Témoin }\end{array}$ & 294 & 0,089 & 7,3 \\
Déchet B solide & 293 & 0,108 & 7,0 \\
$\begin{array}{l}\text { 40-60 cm } \\
\text { Témoin }\end{array}$ & 291 & 0,091 & 7,1 \\
Déchet B solide & 298 & 0,125 & 7,2 \\
\hline
\end{tabular}

Tableau XI. Teneur du sol en différents éléments minéraux et $\mathrm{pH}$ à la récolte du maïs.

\begin{tabular}{|c|c|c|c|}
\hline & $\begin{array}{c}\text { Baryum } \\
\text { total } \\
\left(\text { mg. }^{\prime} g^{-1}\right)\end{array}$ & $\begin{array}{c}\text { Sodium } \\
\text { échangeable } \\
\left(\text { méq. } 100 \mathrm{~g}^{-1}\right)\end{array}$ & $p H$ \\
\hline \multicolumn{4}{|l|}{$0-10 \mathrm{~cm}$} \\
\hline Témoin & 491 & 0,042 & 7,2 \\
\hline Déchet B 317 & 693 & 0,904 & 7,7 \\
\hline \multicolumn{4}{|l|}{$10-20 \mathrm{~cm}$} \\
\hline Témoin & 341 & 0,039 & 7,3 \\
\hline Déchet B 317 & 441 & 0,560 & 8,2 \\
\hline \multicolumn{4}{|l|}{$20-40 \mathrm{~cm}$} \\
\hline Témoin & 268 & 0,037 & 7,9 \\
\hline Déchet B 317 & 485 & 0,203 & 7,9 \\
\hline \multicolumn{4}{|l|}{$40-60 \mathrm{~cm}$} \\
\hline Témoin & 233 & 0,055 & 8,1 \\
\hline Déchet B 317 & 246 & 0,091 & 8,2 \\
\hline
\end{tabular}

L'apport de déchet $B$ a entraîné également une augmentation des teneurs en $\mathrm{Na}$ sur tout le profil compte-tenu de sa mobilité. Elle est plus importante sur maïs (x 20) que sur blé (x 4) dans l'horizon $0-10 \mathrm{~cm}$ et traduit la différence d'apport (151 kg.ha-1 de $\mathrm{Na}$ sur blé et 425 $\mathrm{kg} \cdot \mathrm{ha}^{-1}$ sur maïs). Elle explique l'altération superficielle de la structure du sol des parcelles de maïs observée $15-20$ j après apport à la suite de pluies.

Le $\mathrm{pH}$ de I'horizon $0-20 \mathrm{~cm}$ sous maîs augmente de 0,5 à 0,9 unité sous l'influence des sels d'accompagnement du déchet $B$.

\section{Effet des déchets sur les cultures}

\section{Observations sur blé}

Les déchets sous forme liquide ou solide ne provoquent aucun dommage sur le feuillage du blé au moment de l'apport.

II apparaît un effet légèrement dépressif par rapport au témoin ammonitrate de tous les traitements déchets sur le rendement en grain, significatif pour le traitement déchet B solide (tableau $X I I)$. La teneur du grain en azote est la plus élevée avec le traitement déchet A 200; elle est la conséquence classique d'une surfertilisation azotée. Cette teneur est légèrement inférieure avec les autres traitements mais ne diffère pas significativement de celle du témoin ammonitrate.

Ces effets sur le rendement et les teneurs en azote du grain conduisent à des exportations accrues de $15 \mathrm{~kg}$ d'azote par rapport au témoin 
Tableau XII. Incidence des traitements sur les quantités de blé récoltées et les exportations d'azote par le grain (moyenne de 4 répétitions).

\begin{tabular}{llll}
\hline & $\begin{array}{c}\text { Grain } \\
\left(q . \mathrm{ha}^{-1}\right) \\
(14 \% \text { humid })\end{array}$ & $\begin{array}{c}\text { Azote } \\
(\%)\end{array}$ & $\begin{array}{c}\text { Azote } \\
\text { exporté } \\
\left({\left.\mathrm{kg} . h \mathrm{~h}^{-1}\right)}\right.\end{array}$ \\
\hline & & & \\
\hline Témoin Ammonitrate & $76,2^{\mathrm{a}}$ & $2,07^{\mathrm{b}}$ & $134,5^{\mathrm{b}}$ \\
Déchet A 100 liquide & $74,3^{\mathrm{ab}}$ & $1,97^{\mathrm{b}}$ & $126,0^{\mathrm{c}}$ \\
Déchet A 200 liquide & $74,3^{\mathrm{ab}}$ & $2,34^{\mathrm{a}}$ & $149,0^{\mathrm{a}}$ \\
Déchet B 100 solide & $72,6^{\mathrm{b}}$ & $1,99^{\mathrm{b}}$ & $124,0^{\mathrm{c}}$ \\
Déchet B 100 liquide & $74,3^{\mathrm{ab}}$ & $1,98^{\mathrm{b}}$ & $126,5^{\mathrm{c}}$ \\
& & & \\
Coef variation & $1,8 \%$ & $1,8 \%$ & $2,4 \%$ \\
\hline
\end{tabular}

Les valeurs suivies de la(les) même(s) lettre(s) ne diffèrent pas au seuil $P=0,05$ (Newman et Keuls).

avec le traitement déchet $A 200$, ou réduites de près de $10 \mathrm{~kg}$ avec les 3 autres traitements. Ces effets, bien que faibles, sont significatifs. Par ailleurs les analyses faites sur les grains du traitement déchet $B 100$ solide et du témoin ne montrent pas d'enrichissement significatif en $\mathrm{Ba}$ : respectivement 4 et $2,5 \mathrm{mg} \cdot \mathrm{kg}^{-1}$ de Ba.

L'efficacité azotée des 2 déchets est du même ordre que celle d'une fertilisation équivalente sous forme d'ammonitrate. Cependant, dans le cas du traitement déchet B 100 solide, le léger effet dépressif observé peut être lié à la persistance plus longue des ions nitreux qui perturberaient temporairement le fonctionnement du végétal.

\section{Observations sur maïs}

Au champ. Sur les parcelles ayant reçu le déchet $B$ en couverture $4 \mathrm{j}$ après semis, la levée des plantes a été perturbée : les décomptes réalisés le 19 mai mettent en évidence un retard et des manques, d'autant plus marqués que la dose de déchet est forte (tableau XIII). Le rendement en grains traduit les différences de peuplement observées : il est significativement le plus faible dans les parcelles les moins peuplées. Une compensation significative s'effectue sur la teneur en azote du grain, mais ne suffit pas à égaliser la quantité d'azote exportée par la récolte du témoin. Par ailleurs les analyses faites sur les grains du témoin et des traitements déchet B 190 et déchet B 317 ne montrent pas d'enrichissement en $\mathrm{Ba}$ : toutes les valeurs fluctuent autour de $1 \mathrm{mg} \cdot \mathrm{kg}^{-1}$ de $\mathrm{Ba}$ (moyenne de 3 répétitions par traitement).

En serre. Cet essai nous montre que l'apport des déchets en post-semis, surtout sous forme liquide, s'avère plus dépressif que l'apport une semaine avant la plantation (tableau XIV). L'effet dépressif enregistré sur la production de matière sèche traduit essentiellement la mauvaise germination du maïs, une relation linéaire existant entre le nombre de graines levées et la quantité récoltée, liaison mise en évidence par une analyse de covariance (non présentée).

En une semaine, une partie des nitrites est déjà transformée en nitrate, ce qui explique le peu d'effet (non significatif) des apports en présemis. En post-semis les formes liquides sont immédiatement réparties, entraînant dans l'ensemble du pot et autour des graines une concentration nitreuse élevée ( $80 \mathrm{mg} \cdot \mathrm{kg}^{-1}$ ) largement supérieure au seuil de toxicité que relèvent Goyal et Huffaker (1982), alors que la solubilisation à partir de la poudre a été progressive, la diffusion rapide (d'où des concentrations sans doute faibles) et les conditions d'oxydation favorables.

Tableau XIII. Incidence des traitements sur la levée (25 jours après semis; peuplement moyen du témoin : 74 pieds au $\mathrm{m}^{2}$ ), les quantités de maïs récoltées et les exportations d'azote des grains (moyenne de 4 répétitions).

\begin{tabular}{|c|c|c|c|c|c|}
\hline & \multicolumn{2}{|c|}{ Nombre de pieds } & \multirow{2}{*}{$\begin{array}{l}\text { Grain (q.ha-1) } \\
(15 \% \text { humid) }\end{array}$} & \multirow{2}{*}{$\begin{array}{c}\text { Azote } \\
(\%)\end{array}$} & \multirow{2}{*}{$\begin{array}{c}\text { Azote exporté } \\
\quad\left(\mathrm{kg}_{\mathrm{h}} \mathrm{ha}^{-1}\right)\end{array}$} \\
\hline & $\%$ du témoin & $\%$ ayant 3 feuilles & & & \\
\hline Témoin 180 & $100^{a}$ & 91 & $96,1^{a}$ & $1,47^{b}$ & $120^{a}$ \\
\hline Déchet B 190 & $85^{b}$ & 59 & $85,0^{b}$ & $1,58^{b}$ & $114^{\mathrm{a}}$ \\
\hline Déchet B 317 & $64 c$ & 18 & $66,7^{c}$ & $1,72^{\mathrm{a}}$ & $97^{b}$ \\
\hline Coef variation & $7,5 \%$ & - & $6,0 \%$ & $4,5 \%$ & $8,2 \%$ \\
\hline
\end{tabular}


Tableau XIV. Influence du déchet B sur une culture de maïs en serre (moyenne de 5 répétitions).

\begin{tabular}{|c|c|c|c|c|}
\hline & \multicolumn{2}{|c|}{$\%$ de levée } & \multicolumn{2}{|c|}{ Mat sèche ( $g$ par pot) } \\
\hline & pré-semis & post-semis & pré-semis & post-semis \\
\hline Témoin ammonitrate & 100 & $85^{a b}$ & 8,49 & $7,27^{\mathrm{ab}}$ \\
\hline \multicolumn{5}{|l|}{ Déchet B : } \\
\hline en poudre & 90 & $97^{a}$ & 7,33 & $8,14^{a}$ \\
\hline solution $\mathrm{pH} 12$ & 92 & $77^{a b}$ & 6,96 & $5,99 a b$ \\
\hline solution $\mathrm{pH} 8$ & 97 & $62^{b}$ & 7,26 & $5,25^{c}$ \\
\hline Coef variation & $8,5 \%$ & $21,8 \%$ & $11,9 \%$ & $20,4 \%$ \\
\hline
\end{tabular}

Le déchet $B$ se révèle toxique sur le maïs en germination. Le résultat obtenu en serre nous indique que l'élément toxique est vraisembablement l'azote nitreux, puisque la forme liquide, qui s'avère la plus toxique, est débarrassée de la plus grande partie des sels d'accompagnement. Le maïs rejoint donc les plantes sensibles à l'ion nitreux à la germination (Van den Hende, 1950).

Ces résultats ne permettent pas d'expliquer nettement l'effet dépressif observé sur maïs au champ avec le produit solide grossièrement broyé puisque dans le cas présent, le produit solide, finement broyé, est moins toxique que les produits en solution ou que l'ammonitrate, en post-semis. On peut penser cependant qu'au champ les particules grossières se sont solubilisées au gré de la pluviométrie, irrégulière à cette époque, créant ainsi des zones à forte concentration en ion nitreux dans le lit de semence, zones peu favorable à une activité efficace de la microflore nitrifiante.

\section{CONCLUSIONS}

La plupart des résultats observés lors des apports en sol de culture ou sur plante en végétation de déchets industriels contenant des ions nitreux confirment les observations faites lors de l'utilisation directe de sels nitreux :

- possibilité de dénitrification chimique importante en sol acide. Les produits formés n'ont pas tous été clairement identifiés : des nitrates apparaissent, conduisant à penser à une dismutation; l'absence de $\mathrm{N}_{2} \mathrm{O}$ conduit à penser que l'azote réduit est plutôt sous forme $\mathrm{N}_{2}$;

- perturbations du fonctionnement de la microflore, notamment en milieu acide : arrêt de la ni- trification et stimulation de la respiration et de la minéralisation de l'azote. Ces perturbations sont très liées à la dose appliquée. C'est cet effet dose qui peut également expliquer une partie des effets observés au champ avec le produit grossièrement broyé qui engendrerait des zones à fortes concentrations nitreuses;

- toxicité du nitrite sur le maïs en germination;

- effet de la température sur la vitesse d'oxydation des nitrites, conforme à ce qui est observé avec d'autres phénomènes biologiques se produisant dans le sol. Les basses températures n'ont pas été prises en compte en laboratoire, la stratégie retenue étant d'utiliser ces produits lorsque la température du sol est suffisamment élevée pour permettre une transformation rapide.

II est donc possible d'utiliser le sol pour recycler des déchets du type sels caloporteurs et sels de bain de trempe. L'azote nitreux qu'ils contiennent, et qui représente plus de la moitié de l'azote minéral total, toxique en l'état, peut être valorisé par les plantes après oxydation en ions nitriques. Pour que cette oxydation soit efficace, un certain nombre de conditions doivent être respectées.

La dose d'apport doit être limitée à $50 \mathrm{~kg} \cdot \mathrm{ha}^{-1}$ d'azote nitreux, afin de minimiser les risques de persistance et de lessivage (incidents climatiques après épandage : température basse, forte pluviométrie) liés à un mauvais fonctionnement de la microflore responsable de l'oxydation des nitrites.

Le $\mathrm{pH}$ du sol est un élément important à prendre en compte puisqu'en dessous de 7 une partie des ions nitreux peut être perdue, diminuant l'efficacité azotée des déchets.

Leur utilisation sous forme solide est possible, surtout s'il y a peu ou pas de cations autres que 
$\mathrm{Na}^{+}$et $\mathrm{K}^{+}$(sels caloporteurs) mais elle entraîne une augmentation de la persistance des ions nitreux dans le sol. Une utilisation en solution neutralisée est préférable. La neutralisation diminue l'agressivité de ces produits et permet, dans le cas des sels de bain de trempe, d'éliminer une grande partie d'éléments indésirables après décantation.

La culture sur laquelle sont apportés ces déchets ressort de notre étude comme un élément important. Le blé au stade montaison valorise correctement un tel apport. Le maïs s'est révélé par contre très sensible aux apports en postsemis, démontrant ainsi que pour d'autres plantes que le blé une expérimentation doit préciser doses, stades et modalités d'apport.

Les quantités de sodium épandues demeurent de faible importance pour des apports de déchets calculés sur la base de $100 \mathrm{~kg} \cdot \mathrm{ha}^{-1}$ d'azote, et ne devraient pas conduire à une dégradation importante des propriétés physiques du sol. L'accumulation des éléments-traces et du Ba devra être surveillée comme c'est le cas avec les épandages des déchets en général, surtout du fait de la variabilité de composition des sels de bain de trempe. Une surveillance de l'utilisation de ces produits est de toute façon indispensable puisqu'il s'agit de déchets ayant une valeur fertilisante et non d'engrais. Cette forme de valorisation, moyennant quelques précautions, est certainement préférable au stockage en décharge.

\section{REMERCIEMENTS}

Ce travail a été partiellement financé par l'ANRED, 2 square Lafayette, 49000 Angers. Les auteurs remercient en particulier MG Poireau qui a suivi cette étude.

\section{RÉFÉRENCES}

Anthonisen AC, Loehr AC, Prakasam TBS, Srinath EG (1976) Inhibition of nitrification by ammonia and nitrous acid. J Water Pollut Control Fed 48, 835-852

Bourquin AW (1990) Bioremediation of hazardous waste. Biofutur 93, 24-35

Chapman HD, Liebig GF (1952) Field and laboratory studies of nitrite accumulation in soils. Soil Sci Soc Am Proc 16, 276-282

Charlot G (1966) Les méthodes de la chimie analytique. Masson, Paris

Chaussod R, Nicolardot B, Catroux G (1986a) Mesure routinière de la biomasse microbienne dans les sols par la méthode de fumigation au chloroforme. Sci sol, 201-226

Chaussod R, Nicolardot B, Soulas G, Joannes H (1986b) Mesure de la biomasse microbienne dans les sols cultivés. II. Cinétiques de minéralisation de la matière organique microbienne marquée au carbone 14. Rev Ecol Biol Sol 23, 183-196

Christianson CB, Hedlin RA, Cho CM (1979) Loss of nitrogen from soil during nitrification of urea. Can $J$ Soil Sci 59, 147-154

Germon JC (1985) Le sol, un système épurateur efficace... s'il est bien géré. Rev Palais Découverte 14, $n^{\circ} 133,19-41$

Goyal SS, Huffaker RC (1982) Nitrogen toxicity in plants. In: Nitrogen in crop production (RD Hauck, ed) Am Soc Agron, 97-118

Gerrestsen FC, de Hoop H (1957) Nitrogen losses during nitrification in solutions and in acid sandy soils. Can J Microbiol 3, 359-380

Justice JK, Smith RL (1962) Nitrification of ammonium sulfate in a calcareous soil as influenced by combinations of moisture, temperature and level of added nitrogen. Soil Sci Am Soc Proc 26, 246-250

Lemaire $\mathrm{F}$ (1969) Action des nitrites sur les végétaux. Ann Agron 20, 317-326

Lenglen M (1947) Sur les possibilités d'emploi de nitrites alcalins comme engrais. CR Acad Agric 33, 404-407

Maillard A (1958) Baryum. In: Nouveau traité de chimie minérale IV Groupe II : glucinium, magnésium, calcium, strontium, baryum, radium ( $\mathrm{P}$ Pascal, ed) Masson, 753-760

Nelson DW, Bremner JM (1969) Factors affecting chemical transformations of nitrite in soils. Soil Biol Biochem 1, 229-239

Nelson DW, Bremner JM (1970) Gaseous products of nitrite decomposition in soils. Soil Biol Biochem 2, 203-215

Nicolardot B (1988) Évolution du niveau de biomasse microbienne du sol au cours d'une incubation de longue durée : relations entre la minéralisation du carbone et de l'azote organique. Rev Ecol Biol Sol $25,287-304$

Oke OL (1966) Nitrite toxicity to plants. Nature 212, 528

Pascal P (1956) Azote. In : Nouveau traité de chimie minérale $X$ : azote, phosphore ( $\mathrm{P}$ Pascal, ed) Masson, 7-713

Paul JL, Polle E (1965) Nitrite accumulation related to lettuce growth in a slightly alkaline soil. Soil Sci $100,292-297$

Tyler KB, Broadbent FE, Hill GN (1959) Lowtemperature effects on nitrification in four California soils. Soil Sci 87, 123-129

Van Cleemput O, Baert L (1984) Nitrite: a key compound in $\mathrm{N}$ loss processes under acid conditions? Plant Soil 76, 233-241

Van Den Hende A (1950) Des effets de l'épandage de nitrites sur des cultures en plein champ. Plant Soil $2,223-230$ 